BIOINFORMATION

Discovery at the interfface of physical and biological sciences

\section{Sliding Box Docking: a new stand-alone tool for managing docking-based virtual screening along the DNA helix axis}

\author{
Andrelly Martins-José \\ Departamento de Química, Universidade Federal de Minas Gerais. Belo Horizonte, MG, Brazil. Av. Pres. Antônio Carlos, 6627. \\ Pampulha, 31.270-901; Andrelly Martins-José - Email: andrellym@gmail.com; *Corresponding author
}

Received July 08, 2013; Accepted July 09, 2013; Published August 07, 2013

\begin{abstract}
:
Sliding Box Docking is a program that manages simulations of ligand docking at different defined positions of a three-dimensional DNA structure. The procedure is similar to inverse docking, which is a method that performs docking simulations of a single ligand in the active sites of different targets. Sliding Box Docking manages docking simulations of one ligand into a box that slides along the DNA helix axis in regular steps. For each box position a score is calculated using the separate Autodock Vina software, and the results are automatically plotted. The evaluation of ligand interaction at different DNA locations can highlight the specificity of ligands for different DNA- sequences. When assessing the affinity between ligans AT base pairs, results for docking simulations with a test set that included berenil, distamycin, hoechst 33258, and netropsin were as expected, agreeing well with affinities previously described in the literature.
\end{abstract}

Availability: Binaries are freely available at https://sourceforge.net/projects/slidingboxdocki

Keywords: DNA, Docking tools, software, docking manager

\section{Background:}

Many compounds that bind to the minor groove of DNA or bind as intercalating ligands to DNA have been discovered. Minor groove-binding drugs complement the shape of the groove, whereas intercalation occurs when ligands of an appropriate size and chemical nature insert themselves between DNA base pairs. The minor groove binders cause little distortion of the DNA backbone conformation and intercalating binders are known to cause higher quantities of distortion; however, both drug-binding mechanisms interfere with DNAprotein interaction. Thus, DNA function can be artificially modulated, inhibited, or activated by binding to these drugs molecules instead of the protein. Such activation or inhibition of DNA function can be used to cure or control diseases such as cancer. Experimental and theoretical studies have shown that a large number of these drug molecules can reversibly bind to the minor groove of the AT-rich DNA regions [1, 2]. Using Sliding Box Docking for simulating the interaction of ligands with AT ISSN 0973-2063 (online) 0973-8894 (print)

Bioinformation 9(14): 750-751 (2013) and CG-rich DNA, we can obtain a better understanding of the specificity of these ligands.

\section{Methodology:}

Molecular docking is a method that predicts the preferred orientation of one molecule relative to a second molecule to allow formation of a stable complex. The affinity of the two molecules is evaluated by a scoring function. The docking software simulates the ligands interaction at a specific target site that is defined by a box. The results of docking simulations are best poses for the ligand into the box, and the respective scores of these insertions. The sliding box method allows a box of a constant size to slide along the DNA structure from the bottom to the top (Figure 1A). The size is automatically calculated by the software, and is defined by the number of base pairs. Docking simulations are performed for every possible box location; thus, producing a score for each potential docking site. Sliding Box Docking is a stand-alone application 
implemented in FreePascal, and distributed for the Linux, platform which provides a command line interface. It was developed to work with Autodock Vina [3], a free piece of software separately distributed.

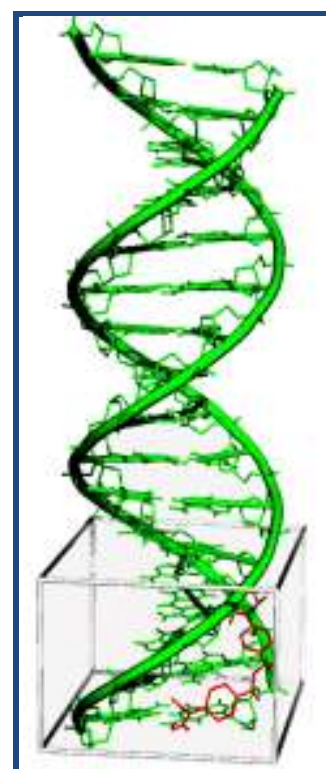

Base pairs: 1 - 4

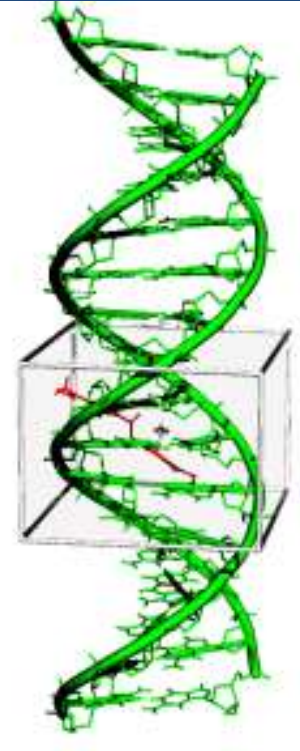

Base pairs: $5 \cdot \mathbf{8}$

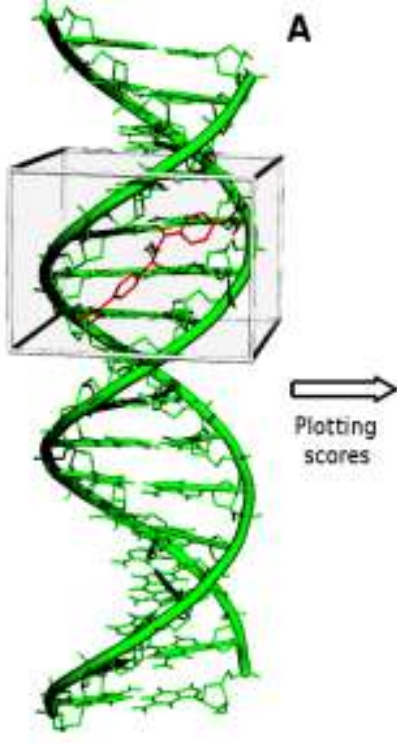

Base pairs: 9 - 12

Figure 1: Schema of positioning box in Sliding Box Docking and compilation of standard results from the screening of four ligands. A) The box slides from bottom to top with constant size. One docking simulation is performed in each position and the values of theoretical free energy are plotted; B) The docking simulations for berenil, distamycin, hoechst 33258, and netropsin into the minor groove of a d(CCGGAATTCGCGATAT) DNA sequence show the best affinity to AT-rich regions.

\section{Input:}

Sliding Box Docking uses a ligand and a three-dimensional DNA structure in Autodock PDBQT format as input. Some three-dimensional DNA structures in PDBQT format are distributed with the software, but other three-dimensional DNA structures can also be used, provided they are in the PDBQT format. The axis of the DNA helical structure should be aligned with the $x, y$ or z-axis, the software automatically detects the axis with which the DNA is aligned. Some threedimensional DNA structures are distributed with the software. The tree-dimensional DNA structures distributed with the software include crystallographics obained from the PDB Data Bank [d(CGCGATATCGCG) and d(CGCGTTAACGCG)] and theoretical structures built using the program Accelrys Discovery Studio [d(CACACTCTGAGAGTGT) and d(CCGGAATTCGCGATAT)] [4].

\section{Output:}

The results of docking simulations are exported in a PNG graphic format and also saved in a text file.

\section{Results \& Discussion:}

Sliding Box Docking has been tested by running it with a ligand dataset that includes berenil, distamycin, hoechst 33258 and netropsin. The literature describes these ligands as specific for AT-rich DNA sequences [5]. A preliminary study showed that selected ligands interact better with the AT-rich position. Docking simulations using Sliding Box Docking showed the expected results for the affinity between ligands and AT base pairs in the target dataset (Figure 1B). The sliding box strategy offers more information than normal DNA-ligand docking simulations because typical DNA-ligand docking simulations show the affinity of the ligand to DNA, but do not show the difference between optimal position and other positions.

\section{Caveat \& Future Development:}

The software has been tested by running it in Ubuntu and is still based on a command-line interface. The software is developed to support Autodock Vina. In the future, I would like to integrate support for other docking tools and include theoretical three-dimensional structures of other DNA sequences into the target data set.

\section{Acknowledgement:}

The authors thank Conselho Nacional de Desenvolvimento Científico e Tecnológico (CNPq) for the financial support for this project.

\section{References:}

[1] Sischka A et al. Biophys J. 2005 88: 404 [PMID: 15516529]

[2] Chaires JB, Biopolymers. 1997 44: 201 [PMID: 9591476]

[3] Trott O \& Olson AJ, J Comput Chem. 2010 31: 455 [PMID: 19499576]

[4] http://accelrys.com/products/discovery-studio/

[5] Abu-Daya A et al. Nucleic Acids Res. 1995 23: 3385 [PMID: 7567447]

Edited by $P$ Kangueane

Citation: Andrelly Martins-José, Bioinformation 9(14): 750-751 (2013) License statement: This is an open-access article, which permits unrestricted use, distribution, and reproduction in any medium, for non-commercial purposes, provided the original author and source are credited 